\title{
Feedforward control of sound transmission using an active acoustic metamaterial
}

\author{
Jordan Cheer, Stephen Daley, Cameron McCormick \\ Institute of Sound and Vibration Research, University of Southampton, Highfield, \\ Southampton, SO17 1BJ
}

\begin{abstract}
Metamaterials have received significant interest in recent years due to their potential ability to exhibit behaviour not found in naturally occurring materials. This includes the generation of band gaps, which are frequency regions with high levels of wave attenuation. In the context of acoustics, these band gaps can be tuned to occur at low frequencies where the acoustic wavelength is large compared to the material, and where the performance of traditional passive noise control treatments is limited. Therefore, such acoustic metamaterials have been shown to offer a significant performance advantage compared to traditional passive control treatments, however, due to their resonant behaviour, the band gaps tend to occur over a relatively narrow frequency range. A similar long wavelength performance advantage can be achieved using active noise control, but the systems in this case do not rely on resonant behaviour. This paper demonstrates how the performance of an acoustic metamaterial, consisting of an array of Helmholtz resonators, can be significantly enhanced by the integration of an active control mechanism that is facilitated by embedding loudspeakers into the resonators. Crucially, it is then also shown how the active acoustic metamaterial significantly outperforms an equivalent traditional active noise control system. In both cases a broadband feedforward control strategy is employed to minimise the transmitted pressure in a one dimensional acoustic control problem and a new method of weighting the control effort over a targeted frequency range is described.
\end{abstract}

Keywords: Active acoustic metamaterial, Active noise control, Feedforward control, Broadband effort weighting 


\section{Introduction}

A significant challenge in noise control engineering is achieving a high level of performance, or noise attenuation, within low size and weight constraints. This challenge becomes particularly demanding at low frequencies where the acoustic wavelength is large and the required dimensions of traditional passive noise control treatments become impractical for many applications. An alternative to increasing the size of the acoustic treatment is to increase its mass, however, this method has similar practical restrictions and it is clear that in many applications, such as the automotive environment, increasing the mass is unacceptable.

One alternative method of controlling low frequency noise that has been extensively investigated, and has relatively recently become a practically viable solution in a number of sectors, is active noise control. In the acoustic domain this technology is based on generating a secondary sound field using control sources that destructively interferes with the primary, unwanted sound field [1]. This technology has been applied in the automotive [2], maritime [3, 4] and aerospace [5, 6] sectors, as well as quite extensively in consumer audio applications [7]. In addition to the benefits afforded by active control technology in terms of the size and weight requirements, there is also a significant benefit in that the system can adapt to changes in the unwanted noise source. Active noise control requires accurate matching of both the spatial and temporal response of the sound field and, therefore, is generally limited to lower frequencies where the acoustic wavelength is long. This makes active noise control a complementary solution to traditional passive noise control treatments, which have large mass or volume requirements when controlling low frequency sound.

In more recent years an alternative approach to achieving noise control at low frequencies has emerged based on the principles of metamaterials developed in the realm of electromagnetics [8]. These so-called acoustic metamaterials use an engineered sub-wavelength structure to achieve significant levels of noise control within low weight and size requirements [9]. In particular, such materials might use a structure consisting of periodically arranged locally resonant elements to introduce band gaps, which are spectral regions in which wave propagation is significantly impeded [10]. For example, an array of split hollow spheres, which act as Helmholtz resonators, has been

Email address: j.cheer@soton.ac.uk (Jordan Cheer) 
used to achieve significant levels of transmission loss over a fixed bandwidth $[11,12,13]$. If an array of identical resonators is employed, the bandwidth over which significant attenuation is achieved is somewhat limited, due to the resonant nature of the band gaps. However, the bandwidth over which attenuation can be achieved can be extended by tuning the resonators in the array to multiple frequencies, although this inherently requires a large number of resonators to be employed and thus begins to reduce the compactness of the metamaterial solution $[13,14]$.

To overcome the limitations of passive acoustic metamaterials, attention has recently been focused on introducing active control technologies into metamaterials designed to control both acoustic and structural waves. In both cases, active metamaterials have the potential to increase the bandwidth of control, overcome some of the losses inherent in a passive metamaterial and facilitate adaptable and tuneable systems [9]. In the field of active acoustic metamaterials, a number of systems have been proposed which use piezoelectric actuation to control wave transmission [15, 16, 17, 18, 19, 20]. In both $[15,16]$ a piezoelectric based metamaterial is used to implement a tuneable acoustic lens, which is capable of steering the direction of wave propagation. In $[17,18,19,20]$ piezoelectric-based metamaterials are used to control acoustic transmission in a waveguide. In particular, [17] proposes an active acoustic metamaterial with a reconfigurable effective density and it is experimentally demonstrated in [18], through measurements of the transmission loss, that this system is able to achieve a band gap with a tuneable frequency. In [19], an array of piezoelectric diaphragms is used to adaptively control the effective density or bulk modulus by utilising a feedback controller between a pressure sensor and the piezoelectric diaphragm. Membrane-based active acoustic metamaterials have also been recently realised using both magnetic [21] and electrical [22] field control mechanisms. These active metamaterials allow the effective density and, therefore, the wave transmission to be tuned by varying either the magnetic [21] or electrical field [22].

In the area of structural vibration control, active metamaterials have also been investigated using perhaps more varied configurations to their acoustic counterparts. In direct analogy to active acoustic metamaterials, a number of systems have been investigated which use shunted piezoelectric elements to directly control structural waves. For example, in [23] shunted piezoelectric patches are used to control wave transmission in a structural waveguide, while in [24] shunted piezoelectric patches are used to create tuneable band gaps in the structural response of a plate. However, active metamaterials have also 
been proposed that, rather than using a purely active mechanism, combine passive and active elements. For example, in [25] a theoretical demonstration of incorporating active 'Skyhook' damping into a metamaterial consisting of periodic resonant masses is presented. More recently, a number of authors have investigated structural active metamaterials which combine an active element into a locally resonant unit. For example, in [26, 27] shunted piezoelectric discs are used to vary the stiffness of local resonators and, therefore, tune their resonance frequency to adjust the stop band. Whereas, in [28] the shear modulus of an electrorheological elastomer is used to tune local resonators in order to steer flexural waves in a plate. Finally, it has been shown in [29] how the width of the band gap in a viscoelastic metamaterial consisting of multiple passive resonators designed for vibration isolation can be increased by incorporating an active feedback control mechanism into the resonating units.

On the basis of the success in the field of structural active metamaterials, some initial work has investigated the potential advantage of incorporating active elements into traditional passive acoustic resonators $[14,30]$. However, this idea has not been experimentally demonstrated or extended to the full metamaterial case, which consists of multiple active resonant units. In this paper the idea of incorporating active control into an acoustic metamaterial consisting of an array of resonant elements is investigated and it is demonstrated that there is a significant performance advantage to be gained by combining passive and active mechanisms rather than using either purely active or passive designs. In Section 2 the acoustic metamaterial is described and its passive performance is presented. In Section 3 the feedforward active control algorithm with frequency dependent control effort weighting is described in detail. In Section 4 offline simulations using measured responses are used to investigate the performance of the active acoustic metamaterial and compare its performance to a purely active design employing standard loudspeakers. In Section 5 the results of a real-time experimental implementation of both the active acoustic metamaterial and the standard active control system are presented. Finally, in Section 6 the results are summarised and conclusions are drawn.

\section{An Active Acoustic Metamaterial}

To overcome the performance limitations of passive metamaterials based on using arrays of Helmholtz resonators [12, 13, 31, 32], as discussed in the in- 
troduction, an active acoustic metamaterial is proposed which incorporates a loudspeaker into the cavity of each of the Helmholtz resonators. Previously, single active Helmholtz resonators have been proposed that incorporate a loudspeaker within the cavity of the resonator [33] and this basic approach is extended here. For the metamaterial application, where multiple active resonators are ultimately required, a standard loudspeaker has been incorporated into a Helmholtz resonator by mounting it at the rear of the resonator's cavity as illustrated in Figure 1. The loudspeaker utilised in the work reported here was a micro-speaker with a fundamental resonance frequency at around $560 \mathrm{~Hz}$ and the dimensions of the speaker and the resonator are also shown in Figure 1. The resonant frequency of the loudspeakers was chosen to be below the Helmholtz resonance frequency, so that the loudspeakers operate in their mass-controlled region and, therefore, have approximately constant acoustic output against frequency. At resonance, the loudspeakers are more efficient and, therefore, operating in this region could improve the overall efficiency of the active control mechanism in practice. Figure 2 shows two photographs of the practical implementation of the active resonator. Figure $2 \mathrm{a}$ shows the assembled resonator and Figure $2 \mathrm{~b}$ shows the two main parts of the resonator separated. From Figure $2 \mathrm{~b}$ it is possible to see the position of the loudspeaker mounted at the rear of the Helmholtz resonator cavity. It is important to highlight that by removing the pipe section on the left of Figure 2b to leave the loudspeaker coupled to an open cavity, as shown on the right of Figure 2b, it is possible to assess the performance of the active system without the effect of the passive resonator - i.e. a standard loudspeaker unit.

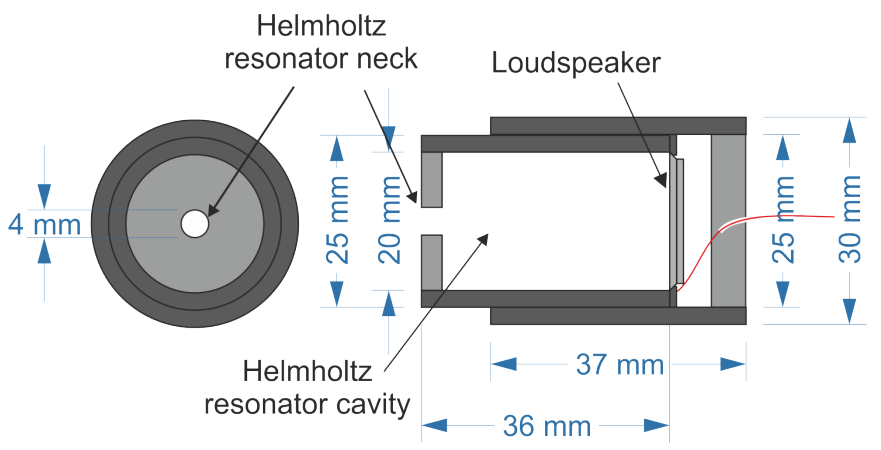

Figure 1: Geometry of the Helmholtz resonator with an embedded loudspeaker. 


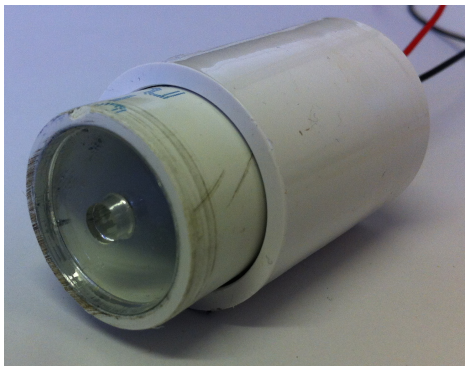

(a)

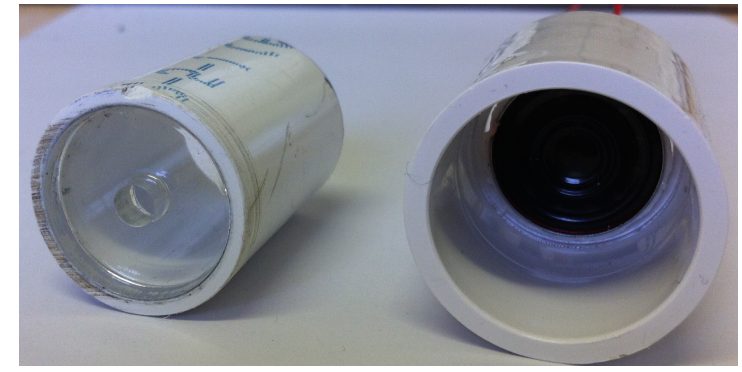

(b)

Figure 2: The practical Helmholtz resonator with an embedded loudspeaker. (a) The constructed resonator; (b) The two main sections of the resonator.

\subsection{Open-Loop Performance}

The performance of an array of standard, passive Helmholtz resonators has previously been investigated $[11,12,13,14]$. However, it is important to confirm that the introduction of the loudspeaker into the resonator does not affect this performance in terms of the level of transmission loss, the resonance frequency or the effective bulk modulus. Therefore, the transmission coefficient of both the open-loop active Helmholtz resonator (i.e. with no voltage driving the loudspeaker) and the passive Helmholtz resonator (i.e. with the loudspeaker removed) has been measured in an impedance tube. It should be noted that when the loudspeaker is removed, it is replaced with a rigid end-cap to ensure that the volume of the resonator cavity remains unchanged. The transmission coefficient has been measured using the fourmicrophone method described in [34]. This method allows the transfer matrix of a material to be determined by generating a plane wave in an impedance tube, which can be achieved using the disturbance loudspeaker shown in Figure 3, and measuring the pressures at four microphone locations, which are also shown in Figure 3. Once the transfer matrix has been calculated from these four pressure measurements, as described in [34], it is then possible to not only calculate the reflection and transmission coefficients, but also the wavenumber and acoustic impedance. Using these parameters, it is then possible to calculate the effective material properties [34], which are integral to evaluating the behaviour of metamaterials.

Figure 4 shows the measured transmission coefficient and the real part of the effective bulk modulus normalised to the bulk modulus of air when 1 , 2, 3 and 4 of either the open-loop, uncontrolled active resonators, Figures 


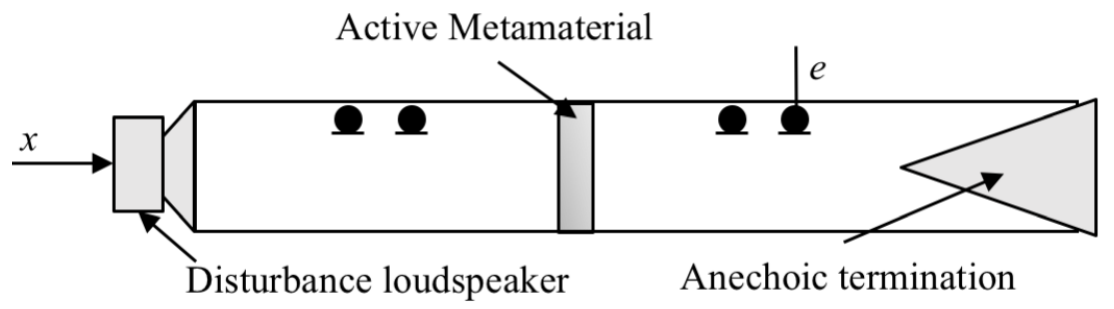

Figure 3: The impedance tube setup showing the four measurement microphones positioned either side of the test material according to the method defined in [34], the reference signal, $x$ and the error signal $e$, the location of the disturbance loudspeaker and the active acoustic metamatierial.

$4 \mathrm{a}$ and $4 \mathrm{~b}$, or the passive resonators, Figures $4 \mathrm{c}$ and $4 \mathrm{~d}$, are installed in the impedance tube. From these plots it can be seen that the band gap, or the region of reduced transmission, is at around $620 \mathrm{~Hz}$ in both cases and similar levels of transmission loss are achieved for the open-loop resonators with embedded loudspeakers and the standard passive resonators without loudspeakers. This demonstrates that the integration of the loudspeaker into the Helmholtz resonator does not have a significant impact on either the resonant frequency, or the level of transmission loss when the control system is open-loop and, therefore, the loudspeaker is not being driven. As expected, increasing the number of resonators in both cases increases the level of transmitted wave attenuation, such that with 4 resonators a transmission loss of around $4 \mathrm{~dB}$ is achieved and the $3 \mathrm{~dB}$ bandwidth is around $70 \mathrm{~Hz}$ in both cases. It can also be seen that the number of resonators influences the normalised effective bulk modulus and this becomes negative between 595 and $685 \mathrm{~Hz}$ in the four resonator case.

\section{Optimal Multichannel Broadband Active Control}

Previous work has demonstrated the potential benefit of incorporating an active control mechanism into a viscoelastic passive metamaterial in order to enhance its performance $[14,25,29]$. To investigate the potential of the active Helmholtz resonator shown in Figure 2a to increase the performance of the passive acoustic metamaterial, it is first necessary to determine a suitable control strategy. In order to understand the physical limitations of the active acoustic metamaterial, rather than the limitations of the controller, an optimal multi-input, multi-output (MIMO) feedforward control strategy will be 


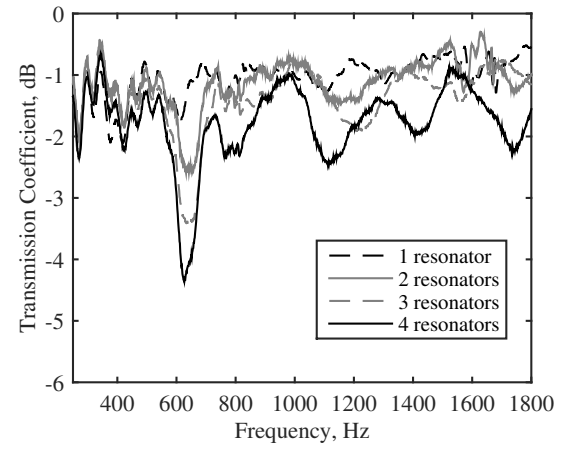

(a) Transmission coefficient for the open-loop active Helmholtz resonators

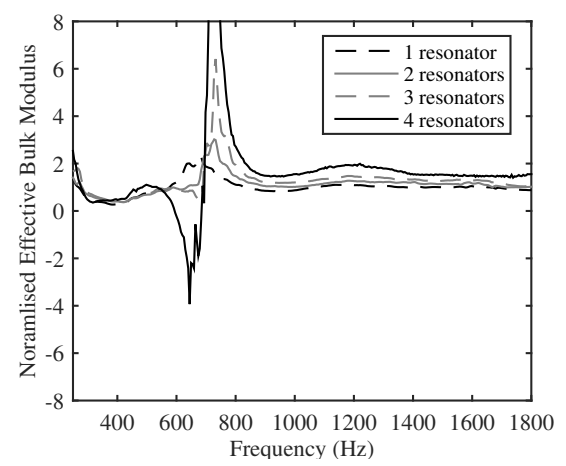

(b) Effective bulk modulus for the openloop active Helmholtz resonators

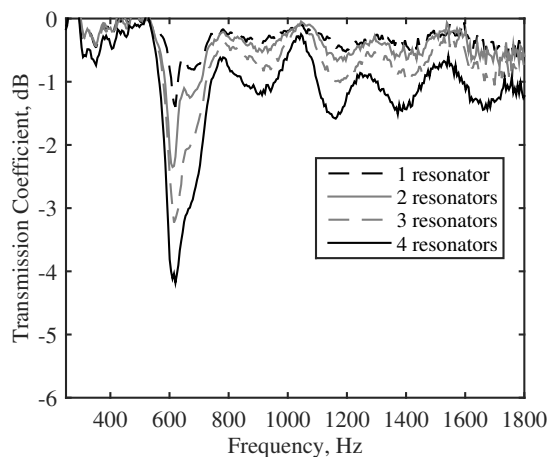

(c) Transmission coefficient for the passive Helmholtz resonators

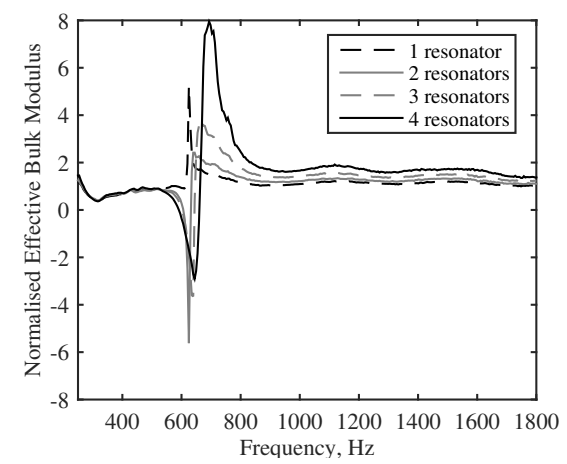

(d) Effective bulk modulus for the passive Helmholtz resonators

Figure 4: The transmission coefficient and normalised effective bulk modulus measured in the impedance tube for 1, 2, 3 and 4 open-loop active Helmholtz resonators (a, b) and the passive Helmholtz resonators (c, d). 
employed to investigate the performance of the system through offline simulations using measured responses. These offline performance predictions will then be validated by employing a practical adaptive controller to implement the system in real-time.

Figure 5 shows the standard MIMO feedforward control architecture [35], where $x$ is the reference signal, $\mathbf{P}$ is the single-input $L$-output primary path response, $\mathbf{G}$ is the $M$-input $L$-output MIMO plant response, $\mathbf{W}$ is the singleinput $M$-output control filter and $\mathbf{e}$ is the vector of $L$ error signals. In the context of the active metamaterial implemented in the impedance tube, the error signal is provided by a microphone located downstream from the active metamaterial and the reference signal is provided by the signal driving the disturbance loudspeaker in the impedance tube, as shown in Figure 3. If it is assumed that the plant and the control filter are linear time invariant and that the control filter is implemented as a bank of $M$ finite impulse response (FIR) filters with $I$ coefficients, then the error signal can be expressed as [35]

$$
\mathbf{e}(n)=\mathbf{d}(n)+\mathbf{R}(n) \mathbf{w},
$$

where $n$ is the sample time, $\mathbf{d}$ is the vector of $L$ disturbance signals measured at the $L$ error sensors, $\mathbf{R}$ is the matrix of reference signals filtered by the plant responses and $\mathbf{w}$ is the vector of control filter coefficients. When the control filter is adaptive, this representation assumes that the filter coefficients are changing slowly compared to the dynamic response of the plant [35].

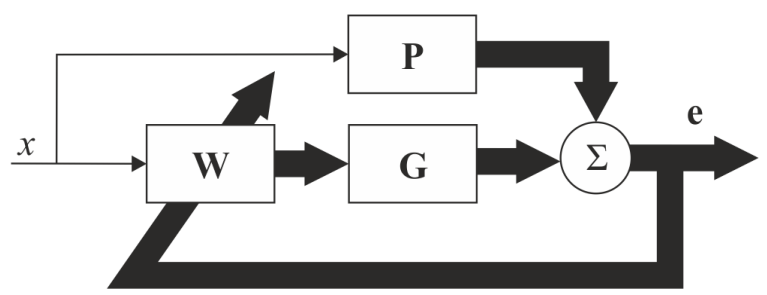

Figure 5: Feedforward active control block diagram.

\subsection{Broadband Optimal Control}

In the case of a broadband multichannel active controller, the control filters are most commonly optimised to minimise the expectation of the sum of the squared error signals. The cost function in this case is given by

$$
J=\mathrm{E}\left[\mathbf{e}^{T}(n) \mathbf{e}(n)\right]
$$


where $\mathrm{E}$ is the expectation operator. In many practical applications, in order to improve the robustness of the algorithm and to limit the magnitude of the control signals, it is common practice to also include a term in the cost function that is proportional to the sum of the squared filter coefficients, or the control effort. This leads to the cost function given by

$$
J=\mathrm{E}\left[\mathbf{e}^{T}(n) \mathbf{e}(n)\right]+\beta \mathbf{w}^{T} \mathbf{w}
$$

where $\beta$ is a positive scalar used to weight the importance of limiting the control effort. Substituting eq. 1 into eq. 3 and expanding gives the cost function as

$$
J=\mathbf{w}^{T} \mathrm{E}\left[\mathbf{R}^{T}(n) \mathbf{R}(n)\right] \mathbf{w}+2 \mathbf{w}^{T} \mathrm{E}\left[\mathbf{R}^{T}(n) \mathbf{d}(n)\right]+\mathrm{E}\left[\mathbf{d}^{T}(n) \mathbf{d}(n)\right]+\beta \mathbf{w}^{T} \mathbf{w} .
$$

The optimal vector of FIR filter coefficients can then be calculated and is given by [35]

$$
\mathbf{w}_{\text {opt }}=-\left\{\mathrm{E}\left[\mathbf{R}^{T}(n) \mathbf{R}(n)+\beta \mathbf{I}\right]\right\}^{-1} \mathrm{E}\left[\mathbf{R}^{T}(n) \mathbf{d}(n)\right] .
$$

In practice, the regularisation coefficient, $\beta$, can be tuned to limit the overall broadband control effort. However, in many practical applications it is often necessary to limit the control effort used by individual control sources and in particular frequency bands. For example, in order to avoid stroke saturation in inertial actuators it may be necessary to limit the low frequency control effort. In the present active metamaterial application it may be desirable to focus the control effort in the bandwidth around the Helmholtz resonance in order to enhance the control, or to weight the control effort so that it broadens the band gap. In the following section a modification to the regularised filtered-reference solution given by eq. 5 will be introduced.

\subsection{Optimal Control with Frequency Dependent Control Effort Weighting}

Frequency dependent control effort weighting can be implemented by defining a frequency weighted control effort as

$$
\mathbf{w}_{b}^{T} \mathbf{w}_{b},
$$

where $\mathbf{w}_{b}$ is the vector of control filter coefficients filtered by an $N_{b}$ coefficient FIR weighting filter $\mathbf{b}$, which can be expressed as the convolution

$$
\mathbf{w}_{b}=\mathbf{b} * \mathbf{w} .
$$


This frequency dependent control effort weighting method was proposed in [36] in the context of calculating fixed optimal filters for sound reproduction systems and has also been employed in the context of personal audio systems [37], however, it has not previously been applied to an active noise control application.

Using the frequency weighted control effort given by eq. 6, a new cost function can be defined as

$$
J_{b}=\mathrm{E}\left[\mathbf{e}^{T}(n) \mathbf{e}(n)\right]+\mathbf{w}_{b}^{T} \mathbf{w}_{b}
$$

In order to derive the optimal vector of control filter coefficients, w, that minimises this cost function, it is first necessary to express the convolution in eq. 7 using the convolution matrix, $\mathbf{B}$, which leads to the matrix-vector multiplication

$$
\mathbf{w}_{b}=\mathbf{B} \mathbf{w} \text {. }
$$

The convolution matrix $\mathbf{B}$ is formed from the coefficients of the weighting filter, $\mathbf{b}$, arranged in the Toeplitz form as

$$
\mathbf{B}=\left[\begin{array}{ccc}
b(0) & 0 & 0 \\
\vdots & \ddots & 0 \\
b\left(N_{b}-1\right) & \ddots & b(0) \\
0 & \ddots & \vdots \\
0 & 0 & b\left(N_{b}-1\right)
\end{array}\right]
$$

This convolution matrix has $I$ columns and $\left(I+N_{b}-1\right)$ rows and, therefore, its sparsity is determined by the difference between $N_{b}$ and $I$.

Using eq. 9 , the cost function given by eq. 8 can be written as

$$
J_{b}=\mathrm{E}\left[\mathbf{e}^{T}(n) \mathbf{e}(n)\right]+\mathbf{w}^{T} \mathbf{B}^{T} \mathbf{B} \mathbf{w}
$$

and substituting eq. 1 for the error leads to

$$
J_{b}=\mathbf{w}^{T} \mathrm{E}\left[\mathbf{R}^{T}(n) \mathbf{R}(n)+\mathbf{B}^{T} \mathbf{B}\right] \mathbf{w}+2 \mathbf{w}^{T} \mathrm{E}\left[\mathbf{R}^{T}(n) \mathbf{d}(n)\right]+\mathrm{E}\left[\mathbf{d}^{T}(n) \mathbf{d}(n)\right] .
$$

The optimal vector of control filter coefficients can then be calculated as

$$
\mathbf{w}_{o p t}=-\left\{\mathrm{E}\left[\mathbf{R}^{T}(n) \mathbf{R}(n)+\boldsymbol{\beta}\right]\right\}^{-1} \mathrm{E}\left[\mathbf{R}^{T}(n) \mathbf{d}(n)\right],
$$

where $\boldsymbol{\beta}=\mathbf{B}^{T} \mathbf{B}$. By designing the FIR weighting filter, $\mathbf{b}$, it is possible to introduce frequency dependent effort weighting and, therefore, focus the control effort in a particular bandwidth. 


\section{Offline Predictions of the Active Acoustic Metamaterial Perfor- mance}

Using the feedforward control strategy with frequency dependent control effort weighting, the performance of the active acoustic metamaterial consisting of different numbers of active Helmholtz resonators has been predicted using the electroacoustic responses measured in a standard impedance tube, as depicted in Figure 3. For example, Figure 6 shows the four active Helmholtz resonators positioned in the impedance tube. From this figure it can be seen that the individual units are positioned in a plane, and thus act on the incident wave in parallel. This is consistent with some previous studies into passive resonator-based acoustic metamaterials [12, 13], but also contrasts with some other configurations in which the individual units have been positioned back-to-back and so act in series [38, 39]. However, because the system considered here is operating in the long wavelength limit, the orientation will not have a significant impact on the performance in this instance. In addition to the closed-loop performance of the active acoustic metamaterial, the performance of an active system using standard loudspeakers without the contribution of the Helmholtz resonator has also been tested. In the first section the regularisation matrix, $\boldsymbol{\beta}$, has been defined to maximise the performance in the bandwidth around the frequency of the Helmholtz resonance, while in the second section the control effort weighting filter has been designed to increase the bandwidth of control. In all cases the active strategy has been optimised to minimise the downstream pressure and, therefore, minimise the transmitted wave; however, in order to provide additional insight and relate the results to previous work on resonator-based

metamaterials, the resulting change in the effective bulk modulus has also been presented.

\subsection{Enhancing the level of performance}

In this section, the feedforward active control strategy has been designed to focus the control effort around the $620 \mathrm{~Hz}$ resonance of the Helmholtz resonators used in the metamaterial. This has been achieved by designing the weighting filter, $\mathbf{b}$, to limit the control effort below $400 \mathrm{~Hz}$ and above $800 \mathrm{~Hz}$. The magnitude response of the resulting control effort weighting filter is shown by the solid black line in Figure 7. Additionally, a standard broadband regularisation factor has been included to ensure that all of the loudspeakers are operating within their linear dynamic range in each case. 


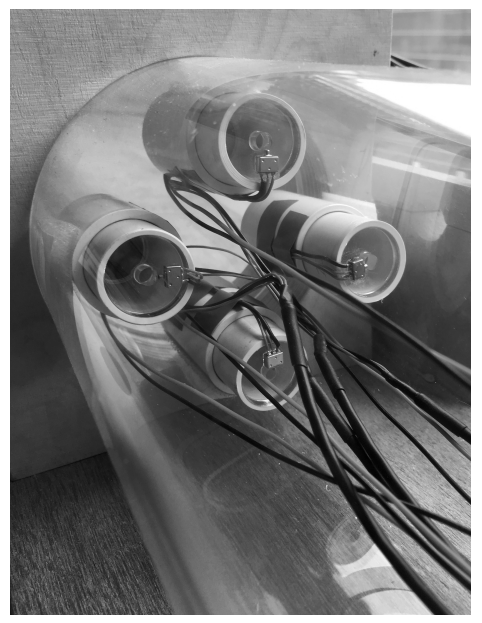

Figure 6: The four active Helmholtz resonators positioned in the impedance tube.

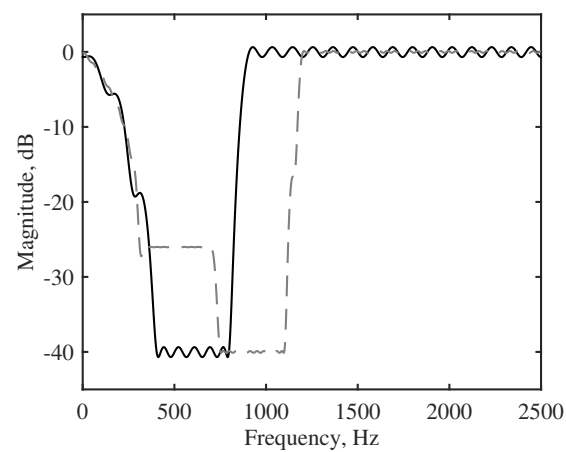

Figure 7: The magnitude response of the control effort weighting filter used to enhance the level of performance (-) and used to enhance the performance bandwidth (- -). 
Figure 8 shows the predicted transmission coefficient and effective bulk modulus of the active acoustic metamaterial with different numbers of resonators, along with the performance achieved by different numbers of standard loudspeakers. Figure 8a shows the transmission coefficient when 1, 2, 3 and 4 active Helmholtz resonators are installed in the impedance tube and optimised to minimise the pressure downstream of the material. Comparing this plot to the passive results shown in Figure 4 it can be seen that the active control mechanism effectively increases both the depth and the bandwidth over which the transmitted wave is attenuated. For example, when using 4 active resonators the transmission loss is up to around $14 \mathrm{~dB}$, which is a $10 \mathrm{~dB}$ increase compared to the open-loop and, therefore, passive case. Additionally, a transmission loss of more than $3 \mathrm{~dB}$ is achieved over a bandwidth of $390 \mathrm{~Hz}$ in the 4 resonator closed-loop active system, compared to a bandwidth of just $70 \mathrm{~Hz}$ in the open-loop or passive system. Additionally, although the closed-loop active system has been optimised to control the transmitted wave and not the material properties, Figure $8 \mathrm{c}$ shows the real part of the effective bulk modulus normalised to the bulk modulus in air. From this plot it can be seen that as the number of resonators is increased, a negative effective bulk modulus is achieved over a wider bandwidth. In particular, for the 4 resonator closed-loop active system a negative effective bulk modulus is achieved between 323 and $840 \mathrm{~Hz}$, which is a 5.7 times increase in the bandwidth over which negativity is achieved compared to the open-loop or passive resonators.

It is evident from these results that the increase in performance achieved by incorporating an active mechanism into the Helmholtz resonator based metamaterial is significant, however, in order to demonstrate the benefits of combining the active and passive elements, Figures $8 \mathrm{~d}$ and $8 \mathrm{f}$ show the transmission coefficient and normalised effective bulk modulus when active control is implemented without the Helmholtz resonators. This has been achieved by removing the front sections of the active units, as shown in Figure 2b. It should be highlighted that, as shown in Figures $8 \mathrm{~b}$ and 8e, the two active configurations have been constrained to use similar levels of control effort over the same bandwidth. From Figure $8 d$ it can be seen that without the Helmholtz resonators, the system achieves a much lower level of transmission loss in the bandwidth of interest. More interestingly, however, it can be seen that the increase in the transmission loss achieved by incorporating the Helmholtz resonators into the active system is greater than the passive performance of the resonators alone; that is, for the system employing 4 


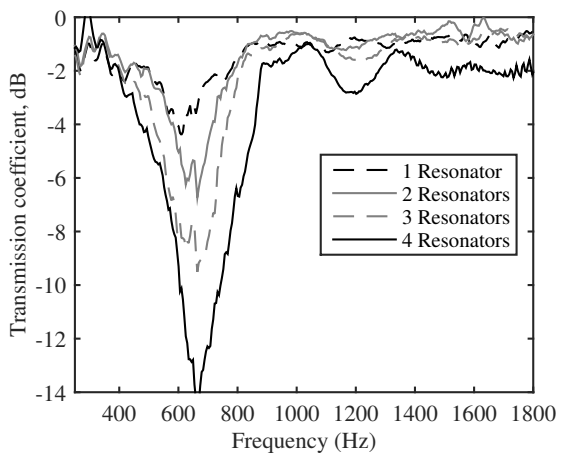

(a) Transmission coefficient for different numbers of active resonators

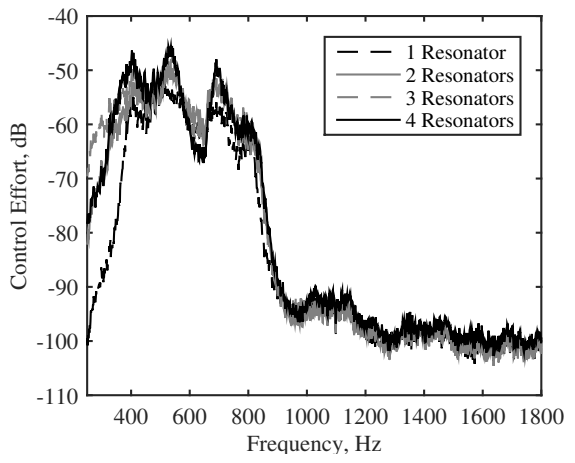

(b) Control effort for different numbers of active resonators

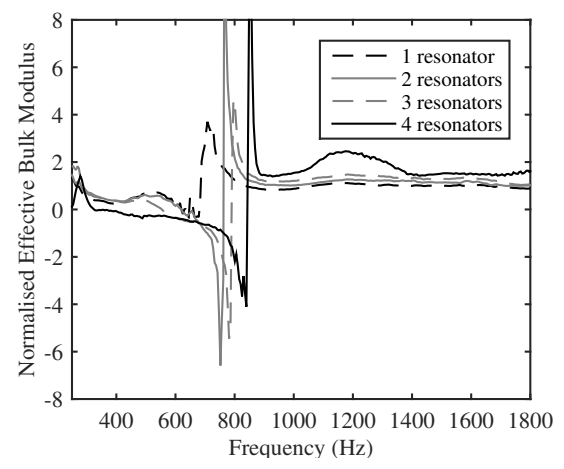

(c) Effective bulk modulus for different numbers of active resonators

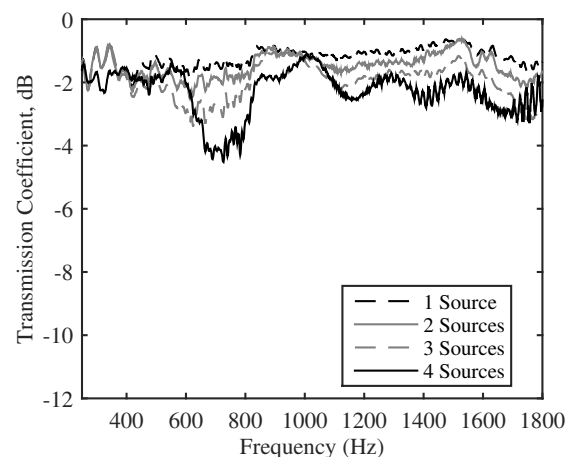

(d) Transmission coefficient for different numbers of standard loudspeakers

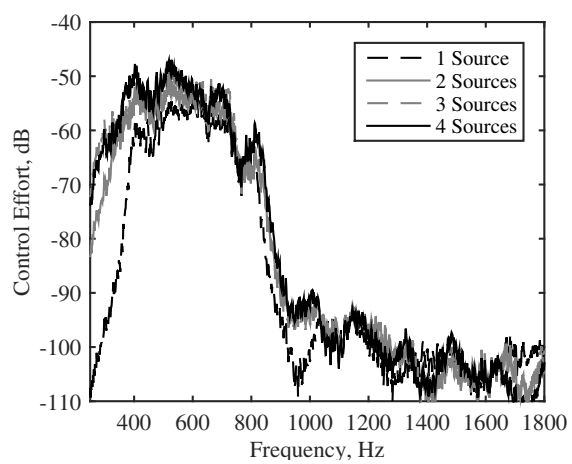

(e) Control effort for different numbers of standard loudspeakers

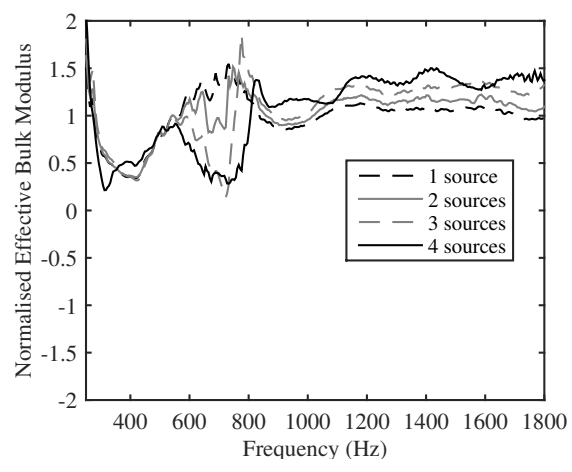

(f) Effective bulk modulus for different numbers of active resonators

Figure 8: The transmission coefficient (a, d), control effort (b, e) and normalised effective bulk modulus (c, f) for different numbers of active resonators (a, b, c) and standard loudspeakers (d, e, f). 
units, incorporating the resonators into the active system provides a $10 \mathrm{~dB}$ increase in the transmission loss, while the passive transmission loss alone is only $4 \mathrm{~dB}$. It is also interesting to observe from Figure $8 \mathrm{f}$ that the closedloop active system using standard loudspeakers does not achieve a negative effective density.

\subsection{Extending the performance bandwidth}

Although it has been shown in the results above that the active acoustic metamaterial is able to significantly increase the level and bandwidth of control compared to both the passive metamaterial and the equivalent array of standard loudspeakers, it is also interesting to investigate the potential to enhance the performance away from the frequency of the Helmholtz resonance. Therefore, an alternative control effort weighting filter has been designed to focus more control effort above the passive resonance, between 800 and $1100 \mathrm{~Hz}$. The magnitude response of this control effort weighting filter is shown by the dashed grey line in Figure 7. From this plot it can be seen that the control effort in the bandwidth between 400 and $800 \mathrm{~Hz}$ is now limited in comparison to the previous configuration to ensure that more effort is used to control the sound transmission above resonance.

The transmission performance of the closed-loop active acoustic metamaterial with 4 units is shown in Figure 9a. This plot shows the open-loop, or passive metamaterial performance along with the performance achieved by the closed-loop active system when optimised using each of the two control effort weighting filters shown in Figure 7. Figure 9b shows the corresponding control effort for the two active strategies and it should be highlighted that the broadband averaged control effort is equal in both cases. From these results it can be seen that the bandwidth over which the transmission coefficient is controlled by the active acoustic metamaterial can be extended using the control effort weighting strategy, however, increasing the bandwidth beyond the frequency of the Helmholtz resonance whilst maintaining the broadband averaged control effort leads to a reduction in the maximum level of attenuation. Specifically, a transmission loss of more than $3 \mathrm{~dB}$ is now achieved over an increased bandwidth of around $600 \mathrm{~Hz}$, but the maximum level of attenuation, which still occurs at the frequency of the Helmholtz resonance, is $7 \mathrm{~dB}$. Figure 9c shows the normalised effective bulk modulus for the open-loop, or passive, and two active closed-loop 4 resonator systems. From this plot it can be seen that for the closed-loop system with a broader bandwidth of transmission loss, the real part of the bulk modulus is negative for a smaller 
bandwidth between around 575 and $845 \mathrm{~Hz}$. Although the upper frequency at which a negative bulk modulus has been achieved has been increased by $5 \mathrm{~Hz}$, the lower frequency has been significantly increased compared to the narrowband closed-loop system. However, it is important to highlight once again that the active control strategy used here has been optimised to minimise the transmitted wave and, therefore, any changes in the effective bulk modulus are simply a by-product of this control strategy. Nevertheless, the presented results demonstrate the potential of the proposed active acoustic metamaterial to manipulate the effective material properties.

It is also interesting to consider the performance achieved by the array of 4 standard loudspeakers and Figure 9d shows the open-loop transmission performance along with the closed-loop performance achieved by the loudspeaker-based active system optimised using each of the two control effort weighting filters shown in Figure 7. Figure 9e shows the corresponding control effort for the two active strategies and, once again, the broadband averaged control effort is equal in both cases and is also equal to that used by the active resonator-based metamaterial. From these results it can be seen that when the control effort is focused in the 800 to $1100 \mathrm{~Hz}$ bandwidth, the loudspeaker-based system achieves a maximum transmission loss of almost $6 \mathrm{~dB}$ at around $1 \mathrm{kHz}$. This is $2 \mathrm{~dB}$ more attenuation than obtained by the active resonator based metamaterial in the increased bandwidth configuration at this frequency and this can related to the reduced sensitivity of the loudspeaker at frequencies above resonance when it is coupled to the Helmholtz resonator. However, it is also important to consider the bandwidth of control and from the results in Figure 9 it can be seen that the loudspeaker-based system only achieves a transmission loss of more than $3 \mathrm{~dB}$ over a $450 \mathrm{~Hz}$ bandwidth compared to the $600 \mathrm{~Hz}$ bandwidth achieved by the equivalent active resonator based configuration. From these results it remains clear that a significant advantage in terms of the overall control bandwidth can be achieved by integrating an active mechanism into a resonant passive metamaterial compared to employing standard loudspeakers in an active system. For completeness, Figure 9f shows the effective bulk modulus of the loudspeaker-based active systems, and once again, it can be seen that the loudspeaker-based system in the open-loop and both closed-loop configurations does not achieve a negative bulk modulus. 


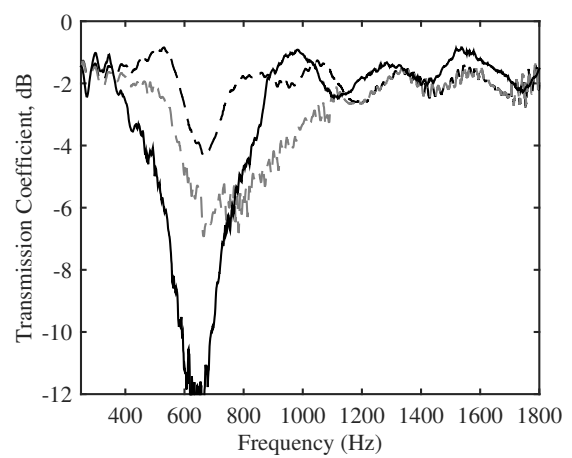

(a) Transmission coefficient for different numbers of active resonators

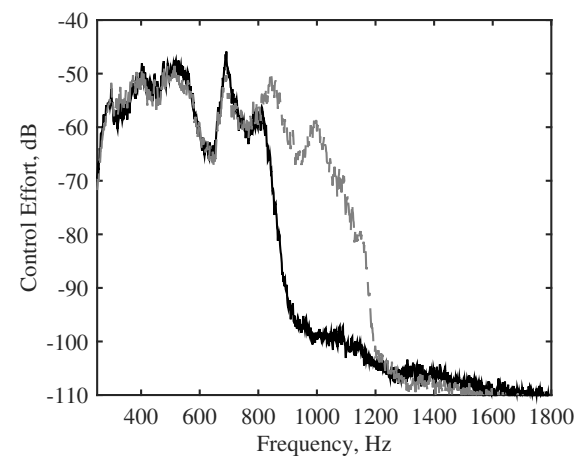

(b) Control effort for different numbers of active resonators

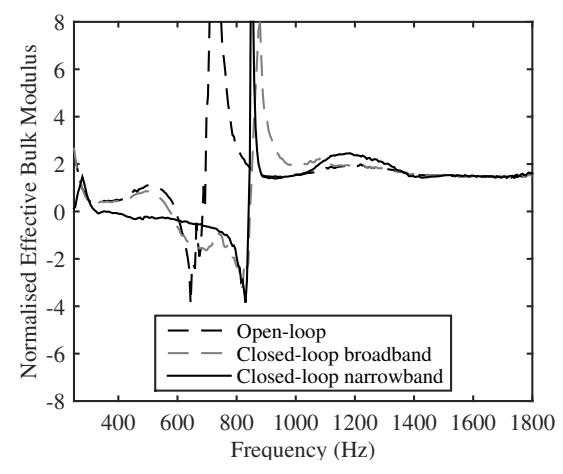

(c) Effective bulk modulus for different numbers of active resonators

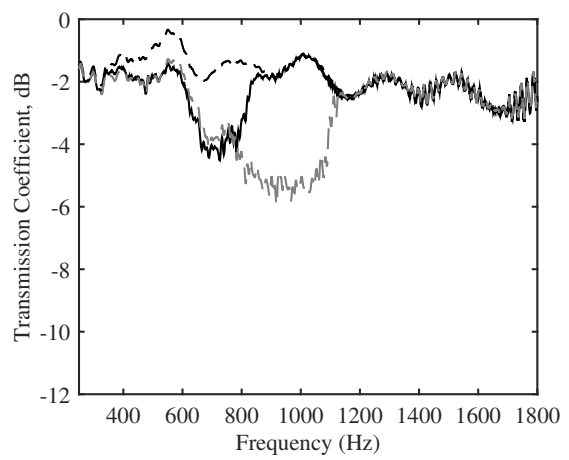

(d) Transmission coefficient for different numbers of standard loudspeakers

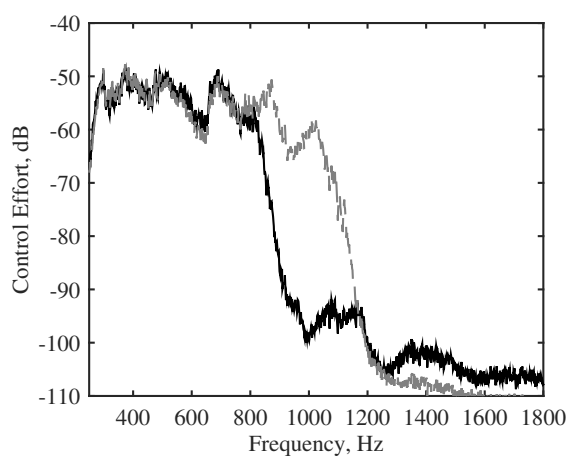

(e) Control effort for different numbers of standard loudspeakers

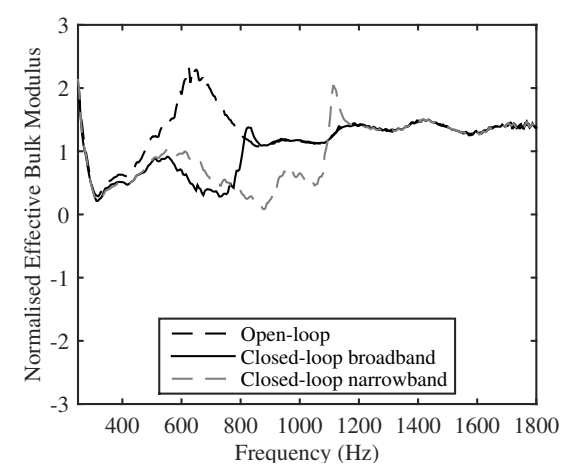

(f) Effective bulk modulus for different numbers of active resonators

Figure 9: The transmission coefficient (a, d), control effort (b, e) and normalised effective bulk modulus (c, f) for four active resonators $(\mathrm{a}, \mathrm{b}, \mathrm{c})$ and four standard loudspeakers (d, e, f). Open-loop performance (black dashed line) and closed-loop performance weighted between 400-800 Hz (black solid line) and 800-1100 Hz (grey dashed line). 


\section{Real-time Adaptive Implementation of the Active Acoustic Meta- material}

In the previous section the performance of the active acoustic metamaterial has been investigated through offline predictions using the measured electroacoustic responses. This has allowed a large number of different configurations to be explored, however, it is important to validate these results experimentally. Therefore, the active acoustic metamaterial and the active system employing standard loudspeakers have both been implemented in real-time using a filtered-reference least mean squares adaptive algorithm [35]. Figure 10a shows the measured transmission coefficient for the active acoustic metamaterial employing 4 units when it is implemented using each of the weighting filters shown in Figure 7; the passive, or open-loop performance is also shown for reference. From these results it can be seen that the measured transmission performance is consistent with the offline predictions shown in Figure 9a and, therefore, these results confirm the potential performance of the active acoustic metamaterial to efficiently control the transmission of sound. In order to also confirm that the active acoustic metamaterial outperforms the active control system employing standard loudspeakers in practice, Figure 10b shows the performance of the standard system measured under the same conditions. It can be seen that these results are consistent with the offline predictions presented in Figure 9d and, therefore, confirm the advantages achieved by integrating an active mechanism into a passive metamaterial. The control effort and effective bulk modulus have not been included for the real-time experimental results for conciseness.

\section{Conclusions}

Acoustic metamaterials that use sub-wavelength, locally resonant elements to achieve high levels of noise control have been of significant interest in recent years. This is mainly due to the potentially high levels of performance that can be achieved within a small and lightweight package. It has been demonstrated in previous work that the performance of such metamaterials can be enhanced by using active control technology, however, this has not previously been fully investigated for an active acoustic metamaterial. This paper has, therefore, presented an investigation into the performance benefits of combining an active control mechanism into a locally resonant metamaterial designed to achieve high levels of transmission loss. 


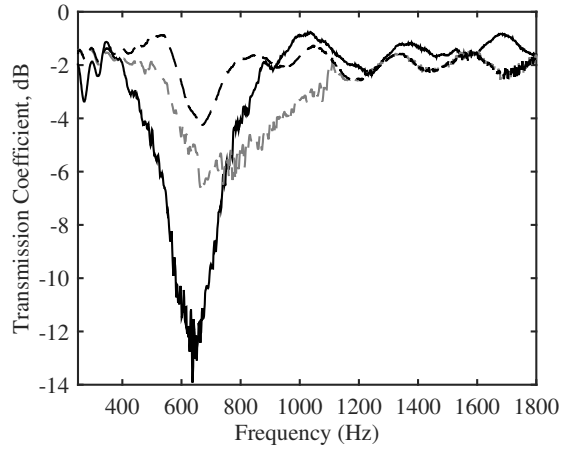

(a) Transmission coefficient measured for the four active resonators

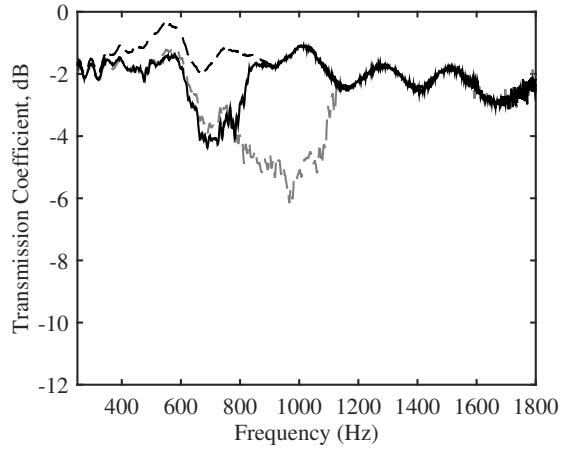

(b) Transmission coefficient measured for the four standard loudspeakers

Figure 10: The transmission coefficient measured for the four active resonators (a) and for the four standard loudspeakers (b) implemented using the adaptive control algorithm. Open-loop performance (black dashed line) and closed-loop active performance weighted between 400-800 Hz (black solid line) and 800-1100 Hz (grey dashed line).

An active acoustic metamaterial has been implemented, which is built up from multiple active Helmholtz resonators, in which a loudspeaker embedded into the resonator facilitates the control mechanism. An optimal feedforward control strategy, which minimises the downstream pressure in an impedance tube, has been used to design the active control filters. In order to focus the control effort in a particular bandwidth, the optimal control filters have been calculated using a frequency dependent regularisation, which has been introduced for the active control application for the first time. It has been shown that by incorporating active control into the locally resonant acoustic metamaterial, a significant increase in the level of transmission loss can be achieved, but it is also possible to broaden the bandwidth over which the transmitted wave is attenuated. For the designed system, this means that a high level of transmission loss can be achieved within a compact solution. It has also been shown that the significant reduction in transmission loss leads to a negative effective bulk modulus and, therefore, although the proposed active metamaterial has been used to control the transmitted wave in this work, it does have the potential to manipulate the effective material properties.

The performance of the active, locally resonant metamaterial has also 
been compared to the equivalent active system using standard loudspeakers when both systems are constrained to operate over a fixed bandwidth and use the same control effort. These results have demonstrated that without the Helmholtz resonators, the loudspeakers do not achieve significant levels of control and, moreover, the increase in performance achieved by incorporating the resonators into the active system is greater than the passive performance of the resonators alone. The presented results have therefore demonstrated the significant advantage that may be achieved by incorporating active elements into a passive, locally resonant acoustic metamaterial.

\section{References}

[1] P. Nelson, S. J. Elliott, Active Control of Sound, Academic Press, London, 1992.

[2] S. J. Elliott, Active noise and vibration control in vehicles, in: X. Wang (Ed.), Vehicle Noise and Vibration Refinement, Woodhead Publishing, Cambridge, 2010, pp. 235-251.

[3] S. Daley, I. Zazas, J. Hatonen, Harmonic control of a 'smart spring'machinery vibration isolation system, Proceedings of the Institution of Mechanical Engineers, Part M: Journal of Engineering for the Maritime Environment 222 (2) (2008) 109-119.

[4] J. Cheer, S. J. Elliott, Active noise control of a diesel generator in a luxury yacht, Applied Acoustics 105 (2016) 209-214.

[5] S. J. Elliott, P. A. Nelson, I. M. Stothers, C. Boucher, In-flight experiments on the active control of propeller-induced cabin noise, Journal of Sound and Vibration 140 (2) (1990) 219-238.

[6] S. A. Lane, R. L. Clark, S. C. Southward, Active control of low frequency modes in an aircraft fuselage using spatially weighted arrays, Journal of Vibration and Acoustics - Transactions of the ASME 122 (2000) 227233.

[7] W. S. Gan, S. M. Kuo, An integrated audio and active noise control headset, Consumer Electronics, IEEE Transactions on 48 (2) (2002) $242-247$. 
[8] D. R. Smith, N. Kroll, Negative refractive index in left-handed materials, Physical Review Letters 85 (14) (2000) 2933.

[9] S. A. Cummer, J. Christensen, A. Alù, Controlling sound with acoustic metamaterials, Nature Reviews Materials 1 (2016) 16001.

[10] Z. Liu, X. Zhang, Y. Mao, Y. Y. Zhu, Z. Yang, C. T. Chan, P. Sheng, Locally resonant sonic materials, Science 289 (5485) (2000) 1734-1736.

[11] C. Ding, L. Hao, X. Zhao, Two-dimensional acoustic metamaterial with negative modulus, Journal of Applied Phsyics 108 (7) (2010).

[12] C.-L. Ding, X.-P. Zhao, Multi-band and broadband acoustic metamaterial with resonant structures, Journal of Physics D: Applied Physics 44 (21) (2011) 215402.

[13] M. Reynolds, S. Daley, An active viscoelastic metamaterial for isolation applications, Smart Materials and Structures 23 (4) (2014) 045030.

[14] M. Reynolds, Active control of viscoelastic metamaterials, Ph.D. thesis, University of Southampton (2015).

[15] B.-I. Popa, D. Shinde, A. Konneker, S. A. Cummer, Active acoustic metamaterials reconfigurable in real time, Physical Review B 91 (2015) 220303.

[16] P. Celli, S. Gonella, Tunable directivity in metamaterials with reconfigurable cell symmetry, Applied Physics Letters 106 (9) (2015).

[17] A. Baz, The structure of an active acoustic metamaterial with tunable effective density, New Journal of Physics 11 (12) (2009) 123010.

[18] W. Akl, A. Baz, Analysis and experimental demonstration of an active acoustic metamaterial cell, Journal of Applied Physics 111 (4) (2012) 044505 .

[19] B.-I. Popa, L. Zigoneanu, S. A. Cummer, Tunable active acoustic metamaterials, Physical Review B 88 (2) (2013) 024303.

[20] B.-J. Kwon, J.-Y. Jung, D. Lee, K.-C. Park, I.-K. Oh, Tunable acoustic waveguide based on vibro-acoustic metamaterials with shunted piezoelectric unit cells, Smart Materials and Structures 24 (10) (2015) 105018. 
[21] X. Chen, X. Xu, S. Ai, H. Chen, Y. Pei, X. Zhou, Active acoustic metamaterials with tunable effective mass density by gradient magnetic fields, Applied Physics Letters 105 (7) (2014).

[22] S. Xiao, G. Ma, Y. Li, Z. Yang, P. Sheng, Active control of membranetype acoustic metamaterial by electric field, Applied Physics Letters 106 (9) (2015).

[23] F. Casadei, T. Delpero, A. Bergamini, P. Ermanni, M. Ruzzene, Piezoelectric resonator arrays for tunable acoustic waveguides and metamaterials, Journal of Applied Physics 112 (6) (2012).

[24] Y. Y. Chen, G. L. Huang, C. T. Sun, Band gap control in an active elastic metamaterial with negative capacitance piezoelectric shunting, Journal of Vibration and Acoustics 136 (6) (2014) 061008-061008.

[25] S. A. Pope, S. Daley, Viscoelastic locally resonant double negative metamaterials with controllable effective density and elasticity, Physics Letters A 374 (41) (2010) 4250-4255.

[26] A. Bergamini, T. Delpero, L. D. Simoni, L. D. Lillo, M. Ruzzene, P. Ermanni, Phononic crystal with adaptive connectivity, Advanced Materials 26 (9) (2014) 1343-1347.

[27] M. A. Nouh, O. J. Aldraihem, A. Baz, Periodic metamaterial plates with smart tunable local resonators, Journal of Intelligent Material Systems and Structures 27 (13) (2016) 1829-1845.

[28] Y. Chen, J. Hu, G. Huang, A design of active elastic metamaterials for control of flexural waves using the transformation method, Journal of Intelligent Material Systems and Structures 27 (10) (2016) 1337-1347.

[29] M. Reynolds, S. Daley, Enhancing the band gap of an active metamaterial, Journal of Vibration and Control (2015) 1077546315600330.

[30] M. Reynolds, S. Daley, Performance and stability constraints of an active acoustic metamaterial, in: Proc. 21st International Congress on Sound and Vibration, Beijing, China, 2014.

[31] C. Boutin, Acoustics of porous media with inner resonators, The Journal of the Acoustical Society of America 134 (6) (2013) 4717-4729. 
[32] L. Schwan, O. Umnova, C. Boutin, H. Shin, S. Taherzadeh, K. Attenborough, Acoustic resonant surface: from nearly-total reflection to nearly-total absorption of sound, in: EuroNoise, 2015, pp. 977-982.

[33] J. Yuan, Active helmholtz resonator with positive real impedance, Journal of Vibration and Acoustics 129 (1) (2007) 94-100.

[34] B. H. Song, J. S. Bolton, A transfer-matrix approach for estimating the characteristic impedance and wave numbers of limp and rigid porous materials, The Journal of the Acoustical Society of America 107 (3) (2000) 1131-1152.

[35] S. J. Elliott, Signal Processing for Active Control, Academic Press, London, 2001.

[36] O. Kirkeby, P. A. Nelson, Digital filter design for inversion problems in sound reproduction, Journal of the Audio Engineering Society 47 (7/8) (1999) 583-595.

[37] M. F. Simon Galvez, S. J. Elliott, J. Cheer, Time domain optimization of filters used in a loudspeaker array for personal audio, Audio, Speech, and Language Processing, IEEE/ACM Transactions on 23 (11) (2015) 1869-1878.

[38] V. M. García-Chocano, R. Graciá-Salgado, D. Torrent, F. Cervera, J. Sánchez-Dehesa, Quasi-two-dimensional acoustic metamaterial with negative bulk modulus, Physical Review B 85 (18) (2012) 184102.

[39] N. Fang, D. Xi, J. Xu, M. Ambati, W. Srituravanich, C. Sun, X. Zhang, Ultrasonic metamaterials with negative modulus, Nature materials 5 (6) (2006) 452-456. 